\title{
Inhibition of the receptor-mediated virion attachment to a lipid membrane
}

\author{
Vladimir P. Zhdanov ${ }^{1,2 *}$ \\ 1 Department of Applied Physics, \\ Chalmers University of Technology, S-41296 Göteborg, Sweden \\ 2 Boreskov Institute of Catalysis, \\ Russian Academy of Sciences, Novosibirsk 630090, Russia
}

Received 05 June 2012; accepted 13 August 2012

\begin{abstract}
:
The forefront of the anti-viral defence is sometimes aimed at virion attachment to a host membrane. This step or, more specifically, virion contacts with cellular membrane receptors (or, e.g., glycolipids) can be inhibited by antibodies (or specially chosen or designed compounds) via their association with virions. In this case, the full-scale attachment of virions to a host membrane occurs via a subtle interplay of the formation and rupture of multiple virion-inhibitor and virion-receptor bonds. We present a kinetic model describing this interplay and illustrating general trends in the process under consideration.

PACS (2008): 87.19.xd, 87.15.K-, 87.10.Ca.

Keywords: $\quad$ virology $\bullet$ viral entry $\cdot$ neutralization $\bullet$ antibody therapeutics $\bullet$ mean-field kinetic equations

(c) Versita sp. z o.o.
\end{abstract}

\section{Introduction}

Viruses contain a DNA or RNA genome protected by a protein capsid and sometimes also by a lipid membrane envelope. Their replication cycles include virion attachment to a host-cell lipid membrane, penetration, uncoating and release of genome, genome replication, viral protein synthesis, capsid assembly, and escape from the host [1, 2]. Every step of this pathway can be targeted for the antiviral host defence. In particular, the forefront of the defence is sometimes aimed at virion attachment to and penetration of a host membrane [3-5]. These steps can be

*E-mail: zhdanov@catalysis.ru inhibited by antibodies (or specially chosen or designed compounds [6, 7]) via their association with virions with no participation of any other components of the immune system. The interpretation of the corresponding experiments has long been focused on the number of antibodies needed to neutralize a virion $[3,8,9]$. The available kinetic models of the first steps of the virion-membrane interaction describe the interplay between virion diffusion and binding to cellular receptors $[10,11]$, fusion and endocytosis of membrane-enveloped virions [12,13], and virion detachment from a membrane $[14,15]$. Models treating in detail the effect of inhibitors on virion attachment to a membrane are still lacking. In this Communication, we introduce and analyze the first model of this category. It scrutinizes the role of various factors in this inhibition and is expected to be useful for researches interested in the correspond- 
ing biophysics and/or biotechnological applications. The model is applicable to the situations occurring in vivo and also to those realized in academic studies (e.g., with attached cells or supported lipid bilayers). In the latter case, comparison of the predictions of the model with the features of experimentally measured kinetics may help to clarify details of the mechanism(s) of the inhibition.

\section{Model}

If a virion has no envelope, its attachment to a lipid membrane usually occurs via the formation of multiple relatively weak bonds between the binding sites at a protein capsid and cellular receptors or, e.g., glycolipids (to be specific, we refer below to cellular receptors). Membraneenveloped virions usually have protein spikes in order to contact a host membrane. An antibody or a specially chosen compound (further referred to as an inhibitor) can bind to the binding sites at the virion surface (i.e., to the sites at a protein capsid or to the protein spikes) and suppress the attachment of virions. The suppression is efficient if the majority of binding sites are blocked. The association of an inhibitor with binding sites is, however, usually reversible, so there is a probability that some of the sites are not blocked. In such cases, a virion can still bind to a membrane via one or two weak bonds. Whether such binding results in the full-scale attachment of a virion depends on the competition between rupture of the virion-inhibitor bonds, and formation and rupture of new virion-membrane bonds. Our goal is to describe the interplay of these processes.

In our model, the distance between the binding sites is considered to be larger than the inhibitor and receptor sizes, and accordingly the cooperative effects in the binding are neglected. In solution (far from the membrane), the inhibitor attachment to and detachment from the binding sites are assumed to be at equilibrium, and accordingly the probability of occupation of a binding site by an inhibitor molecule is described by the conventional equation,

$$
\rho=\frac{\kappa_{\mathrm{a}} c}{\kappa_{\mathrm{d}}+\kappa_{\mathrm{a}} c}
$$

where $\kappa_{\mathrm{a}}, \kappa_{\mathrm{d}}$ and $c$ are the inhibitor attachment and detachment rate constants and concentration, respectively.

If a virion has no envelope, its protein capsid can be viewed as a biological nanoparticle (see, e.g., Fig. 3 below). The interaction or, more specifically, the initial interaction of a virion with a membrane (before appreciable penetration) includes a few binding sites located at one of its facets, and may also include a few sites located at adjacent facets. The initial interaction of a membraneenveloped virion with a membrane includes a few binding sites as well. Focusing on this stage, we consider that the virion binding to the membrane is mediated by $n$ equivalent binding sites located in the virion-membrane contact area.

The attachment of virions to the membrane is considered to be kinetically limited (the diffusion-related corrections can be taken into account if necessary by using the conventional theory of diffusion-limited reactions). In particular, the attachment is considered to be possible if at least one of the $n$ binding sites is vacant. The attachment rate constant is represented as

$$
k_{\mathrm{a}}=k_{\circ} \sum_{i=0}^{n-1} p_{i} \chi_{i} \eta_{i}
$$

where $k_{\circ}$ is the rate constant of the formation of the first virion-receptor bond in the case when all the $n$ binding sites are vacant, $i$ is the number of inhibitors occupying the sites in the beginning (at $t=0$ ) of the virion-membrane contact,

$$
p_{i}=\frac{n ! \rho^{i}(1-\rho)^{n-i}}{i !(n-i) !}
$$

is the probability that $i$ binding sites are occupied by the inhibitor at the beginning of the virion-membrane interaction,

$$
\chi_{i}=(n-i) / n
$$

is the factor taking into account that only $n-i$ vacant sites are available for the formation of the first virionreceptor bond in the beginning, and $\eta_{i}$ is the probability that the formation of the first virion-receptor bond results eventually in the full-scale attachment of a virion.

If $\eta_{i}=1$, i.e., the formation of the first virion-receptor bond is sufficient for the full-scale attachment of a virion, Eq. 2 in combination with Eqs. 3 and 4 yield

$$
k_{\mathrm{a}}=k_{\circ}(1-\rho) .
$$

Using expression 1 for $\rho$, one can rewrite Eq. 5 as

$$
k_{\mathrm{a}}=\frac{k_{\circ} \kappa_{\mathrm{d}}}{\kappa_{\mathrm{d}}+\kappa_{\mathrm{a}} c} .
$$

In reality, the formation of the first virion-receptor bond is often not sufficient for the full-scale attachment of a virion (especially if $\rho$ is close to unity), and we should take into account that $\eta_{i}<1$. To calculate $\eta_{i}$, we use probabilities $P_{l, j}(t)(0 \leq l+j \leq n)$ that at time $t$ after the beginning of the virion-membrane contact, the contact 
area contains $l$ and $j$ binding sites occupied by the inhibitor molecules and membrane receptors, respectively. The equations, used here for these probabilities, are similar to those employed earlier to describe cell adhesion (reviewed in Ref. [16]) and virion detachment (see the supplementary material in Ref. [15]). The only difference is that the latter processes are usually described assuming all the bonds (e.g., the capsid-glycolipid bonds [15]) to be equivalent, while we scrutinize formation of the bonds of two types including the capsid-inhibitor bonds and the capsid-receptor bonds. Although the approach we employ is conceptually simple, the corresponding equations are cumbersome. To simplify the analysis, we take into account that during the virion-membrane contact the formation of new bonds with receptors is often much more probable than the formation of new bonds with the inhibitor (this is the case provided that the concentration, $C$, of receptors is not too low). Under this condition, we neglect the formation of new bonds with the inhibitor. In this case, the type of the equations we should solve is as follows

$$
\begin{array}{rl}
d P_{l, j} / d & t=(l+1) \kappa_{\mathrm{d}} P_{l+1, j}-l \kappa_{\mathrm{d}} P_{l, j} \\
& +(j+1) v_{\mathrm{r}} P_{l, j+1}-j v_{\mathrm{r}} P_{l, j} \\
& +(n-l-j+1) v_{\mathrm{f}} C P_{l, j-1}-(n-l-j) v_{\mathrm{f}} C P_{l, j},
\end{array}
$$

where $v_{f}$ and $v_{r}$ are the rate constants of the formation and rupture of the virion-receptor bonds. Using the equations of this type, we neglect lateral receptor-receptor interaction resulting in correlations in the arrangement of receptors (depending on the value of this interaction and receptor concentration, such correlations may be observed on the length scale from a few $\mathrm{nm}$ to $\mu \mathrm{n}$ ). In other words, we neglect the formation of domains (or rafts) in a membrane (this effect is reviewed in Refs. $[17,18]$ ). This approximation is often reasonable, at least in the case when $C$ is not too high. If necessary, the raft formation can be taken into account analytically (e.g., by employing the quasi-chemical approximation as described in Ref. [15]) or by using the Monte Carlo technique.

In addition, we consider that the formation of new bonds with receptors is fast compared to the rupture of bonds, i.e., $v_{f} C \gg v_{f}$ (and $v_{f} C \gg \kappa_{d}$ ). In this case, the relaxation in the space of the virion-receptor bonds is rapid, and accordingly we consider that these bonds are at the equilibrium corresponding to a given number $(l)$ of the virioninhibitor bonds. Under this condition, we can introduce the virion detachment rate constant, $r_{l}$, corresponding to number $l$, and replace Eqs. 7 by the reduced equations describing the rupture of the virion-inhibitor bonds and the virion detachment. Following this way, we introduce the probability, $P_{l}(t)$, that at time $t$ after the beginning of the virion-membrane contact, the contact area contains $l$ binding sites occupied by the inhibitor. If $i(0 \leq i \leq n-1)$ is the initial number of inhibitors in this area, we have $i+1$ probabilities $P_{l}(t)$ with $0 \leq l \leq i$. The equations for these probabilities with $l=i, 1 \leq l \leq i-1$ and $l=0$ are, respectively, read as

$$
\begin{gathered}
d P_{i} / d t=-i \kappa_{\mathrm{d}} P_{i}-r_{i} P_{i} \\
d P_{l} / d t=(l+1) \kappa_{\mathrm{d}} P_{l+1}-l \kappa_{\mathrm{d}} P_{l}-r_{l} P_{l} \\
d P_{0} / d t=\kappa_{\mathrm{d}} P_{1} .
\end{gathered}
$$

Using these equations, we allow the virion detachment for $1 \leq l \leq i$ and neglect for $l=0$. Physically, this is reasonable if, in the absence of the inhibitor, the virion binding is strong and the virion detachment in this case is either slow, on the time scale of attachment, or becomes impossible due to subsequent steps on the pathway of the virion-membrane interaction.

Integrating Eqs. 8-10 with the corresponding initial conditions,

$$
P_{l}(0)=\left\{\begin{array}{l}
1 \text { for } l=i \\
0 \text { for } 0 \leq l \leq i-1
\end{array}\right.
$$

we obtain

$$
\begin{gathered}
\int_{0}^{\infty} P_{i}(t) d t=\frac{1}{i \kappa_{\mathrm{d}}+r_{i}} \\
\int_{0}^{\infty} P_{l}(t) d t=\frac{(l+1) \kappa_{\mathrm{d}}}{l \kappa_{\mathrm{d}}+r_{l}} \int_{0}^{\infty} P_{l+1}(t) d t \\
P_{0}(\infty)=\kappa_{\mathrm{d}} \int_{0}^{\infty} P_{1}(t) d t
\end{gathered}
$$

The probability that the formation of the first virionreceptor bond results in the full-scale attachment of a virion can be identified with $P_{0}(\infty)$, i.e, $\eta_{i} \equiv P_{0}(\infty)$. Employing this definition and Eqs. 11-14, we get

$$
\eta_{i}=\prod_{l=1}^{i} p_{l}
$$

where

$$
p_{l}=\frac{l \kappa_{\mathrm{d}}}{l \kappa_{\mathrm{d}}+r_{l}} .
$$

The physical meaning of these expressions is straightforward. In each state $l$ (with $l>0$ ), a virion may either reach state $l-1$ or leave the membrane. The probability of a transition to state $l-1$ is given by expression 16 . To reach the fully bound state (with $l=0$ ) from the initial state (with $l=i$ ), a virion should sequentially perform a series of such transitions down to $l=0$, and accordingly the probability to reach the fully bound state is equal to a product of the corresponding probabilities $p_{l}$ (Eq. 15). 

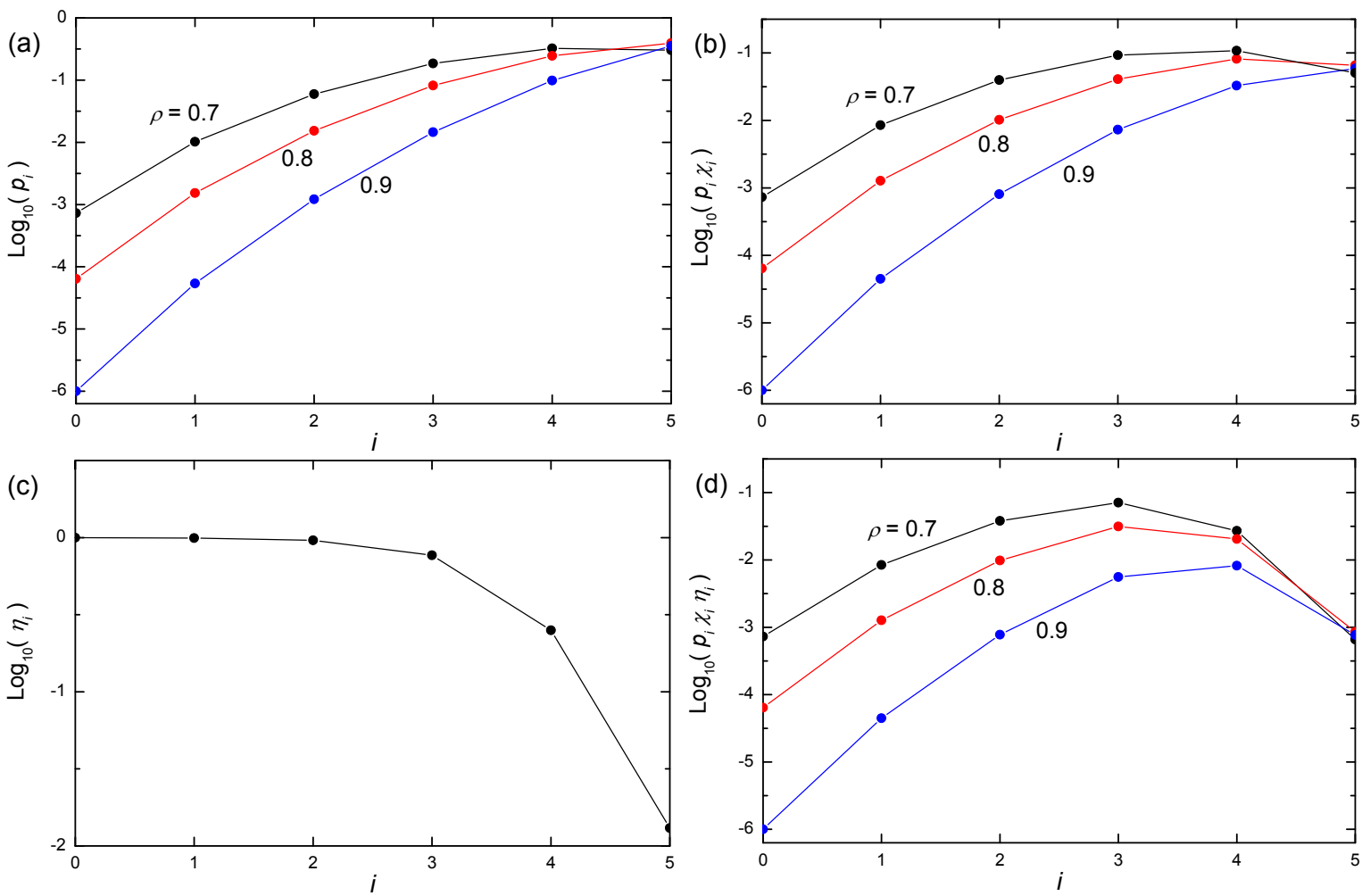

Figure 1. Specification of the contribution of various factors to the normalized attachment rate constant, $k_{\mathrm{a}} / k_{\mathrm{o}}$. This rate constant is determined by a sum of products $p_{i} \chi_{i} \eta_{i}$ (Eq. 2). Panels (a-c) show probabilities $p_{i}$ (Eq. 3), $p_{i} \chi_{i}$ (Eqs. 3 and 4), and $\eta_{i}$ (Eq. 15) as a function of $i$. Panel (d) exhibits the dependence of the whole products of these factors on $i$. The results presented in panels (a-d) have been obtained assuming that the number of binding sites located in the virion-membrane contact area is equal to 6 .

If the number of the virion-inhibitor bonds is $l$, the virion detachment rate constant can be represented as (see Eq. 16 in the main text or Eq. 8 in the supplementary material in Ref. [15])

$$
r_{l}=\frac{r_{*}}{\left(1+\kappa_{\mathrm{f}} C / \kappa_{\mathrm{r}}\right)^{n-l}}
$$

where $r_{*}$ is the departure rate constant corresponding to the state with no virion-receptor bonds. Employing expression 17, we neglect correlations in the arrangement of receptors. As already noticed below Eq. 7, this approximation is often reasonable at least in the case when the concentration of receptors in a membrane is not too high and, if necessary, it can be relaxed.

Eqs. 2-4, and 15-17 allow us to calculate the impact of various parameters on the full-scale virion attachment probability, $k_{\mathrm{a}} / k_{\circ}$.

\section{Model predictions}

Typical predictions of our model are exhibited in Figs. 1 and 2. In particular, taking into account that according to Eq. 2 the normalized attachment rate constant, $k_{\mathrm{a}} / k_{\circ}$ is determined by a sum of products $p_{i} \chi_{i} \eta_{i}$ corresponding to different numbers of inhibitors occupying the sites in the beginning of the virion-membrane contact, we show in Fig. 1(a-c) the dependence of $p_{i}, p_{i} \chi_{i}$, and $\eta_{i}$ on $i$ for $n=6$ (an example of a virion with this $n$ is presented in Fig. 3), $\rho=0.7,0.8$, and $0.9, \kappa_{\mathrm{f}} C / \kappa_{\mathrm{r}}=10$, and $\kappa_{\mathrm{d}} / r_{*}=10^{-3}$. The dependence of the whole products of these factors on $i$ is exhibited in Fig. 1(d). In this case, $\rho$ is appreciable and initially a virion typically has in the contact area 4 or 5 binding sites occupied by the inhibitor (Fig. 1(a)). The states with $i=3-5$, makes the main contribution to the initial contact (Fig. 1(b)). The probability that the formation of the first virion-receptor bond results in the full-scale attachment of a virion is close to unity for $0 \leq$ $i \leq 3$, and appreciably smaller than unity for $i=4$ and especially for $i=5$ (Fig. 1(c)). Due to the interplay of these factors, the main contribution to the rate constant of the full-scale virion attachment to the membrane is given 


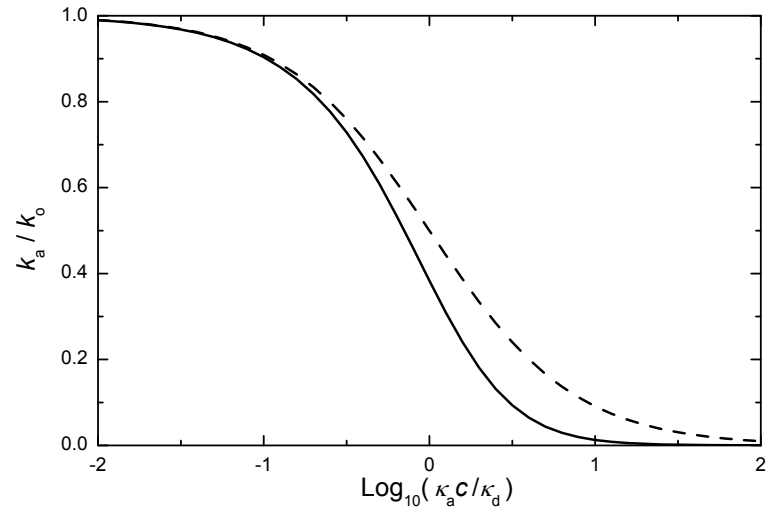

Figure 2. Probability of the full-scale virion attachment as a function of $\kappa_{\mathrm{a}} c / \kappa_{\mathrm{d}}$ according to Eq. 2 with $\eta_{i}<1$ (solid line) and Eq. 6 with $\eta_{i}=1$ (dashed line).

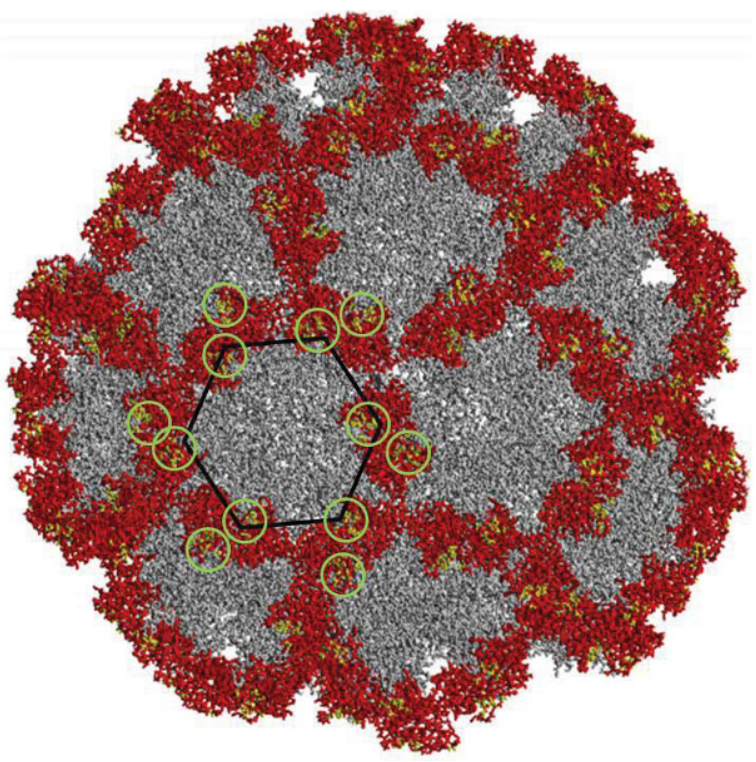

Figure 3. Frontal view of a capsid of norovirus including 45 dimers of the capsid protein (adapted from Ref. [15]). These proteins form facets. The six binding sites on the vertices of a hexagonal facet (this facet can be viewed as a contact area) are indicated by light green circles (these sites, located $\sim 6 \mathrm{~nm}$ apart, are linked by a black line) along with the neighboring binding sites on the same dimers. The latter sites are located $\sim 2 \mathrm{~nm}$ downwards with respect to the hexagonal facet formed by the former sites. Attachment of this virus to a membrane can be inhibited, e.g., by citrate [6] and other compounds [7].

by the state with $i=3$ (Fig. 1(d)).

Physically, the normalized attachment rate constant, $k_{\mathrm{a}} / k_{\circ}$, represents the probability of the full-scale virion attachment. This probability decreases with increasing $\kappa_{\mathrm{a}} c / \kappa_{\mathrm{d}}$ (Fig. 2). The decrease of $k_{\mathrm{a}} / k_{\circ}$, predicted by Eq. 2 (with $\eta_{i}<1$ ), is seen to be somewhat more abrupt compared to that predicted by Eq. 6 (with $\eta_{i}=1$ ).

\section{Conclusion}

Although the results presented in Figs. 1 and 2 have been obtained for specific values of the model parameters, they illustrate general trends in the inhibition of the virion attachment to a lipid membrane. The simplest situation takes place when the formation of the first virion-receptor bond is sufficient for the full-scale attachment of a virion. In this case (with $\eta_{i}=1$ ), the dependence of the attachment rate constant on $c$ is described by a simple expression 6 of the Langmuirian type [19] (in biophysics, such expressions are widely used to describe the ligand-receptor interaction or the function of transcription factors [20]). If $\eta_{i}<1$, i.e., the virion attachment may be followed by detachment, the dependence of the attachment rate constant on $c$ is expected (Fig. 2) to be stronger compared to that predicted by expression 6 . To verify these predictions, one needs to perform accurate measurements of the dependence of this rate constant on $c$. At present, such measurements are lacking.

Finally, we mention possible extensions of the theory presented here. As it stands, the model does not take diffusion limitations into account. Such limitations may be important. Their analysis depends on specific geometry and if necessary can be performed by using the conventional theory of diffusion-limited reactions (see, e.g., general theory [21], recent related articles [10, 11, 22, 23], and references therein). Among other extensions of our analysis, we may refer to the case of attachment of an inhibitor to two binding sites. More complex rules describing the interplay between the formation of capsid-inhibitor and capsid-receptor bonds can also be introduced into the model.

\section{Acknowledgments}

This work was supported by Swedish Research Council (Grant 2010-5063). The author thanks F. Höök, G. Larson, M. Bally, and W. Nasir for useful discussions. W. Nasir is acknowledged also for constructing Fig. 3.

\section{References}

[1] A.J. Cann, Principles of Molecular Virology (Elsevier, Amsterdam, 2012)

[2] J. Mercer, M. Schelhaas, A. Helenius, Ann. Rev. Biochem. 79, 803 (2010)

[3] P.J. Klasse, Q.J. Sattentau, J. Gen. Virol. 83, 2091 (2002)

[4] M. Law, L. Hangartner, Curr. Opin. Immun. 20, 486 (2008) 
[5] J.C. Tilton, R.W. Doms, Antiviral Res. 85, 91 (2010)

[6] G.S. Hansman et al. J. Virol. 86, 284 (2012)

[7] C. Rademacher et al., Chem. Eur. J. 17, 7442 (2011)

[8] C. Magnus, P. Rusert, S. Bonhoeffer, A. Trkola, R.R. Regoes, J. Virol. 83, 1523 (2009)

[9] C. Magnus, R.R. Regoes, PLoS Comput. Biol. 6, e1000713 (2010)

[10] T. Chou, M.R. D’Orsogna, J. Chem. Phys. 127, 105101 (2007)

[11] M.M. Gibbons, T. Chou, M.R. D'Orsogna, J. Phys. Chem. B 114, 15403 (2010)

[12] T. Chou, Biophys. J. 93, 1116 (2007)

[13] S.A. Nowak, T. Chou, Biophys. J. 96, 2624 (2009)

[14] M. Bally et al., Phys. Rev. Lett. 107, 188103 (2011)

[15] M. Bally, K. Dimitrievski1, G. Larson, V.P. Zhdanov, F. Höök, Phys. Biol. 9, 026011 (2012)
[16] C. Zhu, J. Biomech. 33, 23 (2000)

[17] D. Lingwood, V. Simons, Science 327, 46 (2010)

[18] J. Fan, M. Sammalkorpi, M. Haataja, FEBS Let. 584, 1678 (2010)

[19] P.W. Atkins, J. de Paula, Physical Chemistry, Eighth Edition (W.H. Freeman and Co., New York, 2006); Chap. 25

[20] C. Michel, Progr. Biophys. Molec. Biol. 102, 16 (2010)

[21] S.A. Rice, Diffusion-Limited Reactions; Comprehensive Chemical Kinetics, 25 (Elsevier, Amsterdam, 1985)

[22] V.P. Zhdanov, B. Kasemo, Phys. Rev. E 74, 021915 (2006)

[23] V.P. Zhdanov, B. Kasemo, Coll. Surf. B: Biointerf. 76, 28 (2010) 